\title{
The effects of enteral feeding with eicosapentaenoic acid, $\gamma$-linolenic acid and antioxidant vitamins on mechanically ventilated patients with severe sepsis and septic shock: a retrospective analysis of Japanese-descendant patients
}

\author{
Alessandro Pontes-Arruda * 1, Hiroyuki Hirasawa *2
}

\begin{abstract}
Objective: Enteral diets enriched with eicosapentaenoic acid (EPA), $\gamma$-linolenic acid (GLA) and antioxidant vitamins have been demonstrated to improve outcomes in patients with acute lung injury (ALI) and acute respiratory distress syndrome (ARDS). There is ethnic difference in the distribution of genetic polymorphisms, and therefore, the efficacy of nutritional treatment may differ among races. We evaluated the efficacy of the enteral diet enriched with EPA, GLA and antioxidants in a Japanese-descendant population with ARDS secondary to severe sepsis or septic shock through a retrospective assessment of the original database of our previous study. Materials and methods: 18 Japanese-descendant patients were identified from the original study based on the ethnic answer on their clinical research forms. All patients were randomly assigned to two groups either with a diet enriched with EPA, GLA and antioxidant vitamins or with an isonitrogenous and isocaloric control diet, delivered at a constant rate to achieve a minimum of $75 \%$ of basal energy expenditure x 1.3 during a minimum of 4 days and monitored for 28-days. Results: Those who received the study diet experienced significant improvements in oxygenation status, more ventilator-free days $(12.0 \pm 2.6$ vs. $4.2 \pm 1.7, P=0.0362)$ and more ICU-free days $(8.9 \pm 2.2$ vs. $2.6 \pm 1.2, P=0.0348)$. No significant changes were observed in terms of the development of new organ failures and mortality outcomes. Conclusions: In Japanese-descendant patients with severe sepsis or septic shock and requiring mechanical ventilation and tolerating enteral nutrition, a diet enriched with EPA, GLA and antioxidants contributed to better ICU outcomes.

Key words: (1) severe sepsis, (2) fish oil, (3) borage oil, (4) randomized, (5) enteral nutrition
\end{abstract}

\section{Introduction}

Acute lung injury (ALI) and acute respiratory distress syndrome (ARDS) are two serious and potentially lethal types of respiratory failure that develop in critically ill patients ${ }^{1}$. The incidence of these conditions in the United States is currently estimated at 56 to 82 cases per 100,000 person-years, making them much more common than many previous studies have suggested ${ }^{2}$. In fact, the total annual mortality due to ALI / ARDS is similar to that attributed to other more widely recognized conditions such as breast cancer, human immunodeficiency virus (HIV), and acquired immunodeficiency syndrome (AIDS) ${ }^{3)}$.

Both ALI and ARDS are severe forms of refractory hypoxemia due to non-cardiogenic pulmonary edema. Both are associated with an inflammatory process that is usually unrestricted to the lungs, but is often characterized as an uncontrolled state of systemic inflammation causing impaired oxygen delivery and tissue injury throughout the body. In the absence of successful treatment, the degenerative process unleashed by ALI and ARDS eventually leads to multiple organ system failure and death.

Enteral nutrition is gaining importance as an adjuvant therapy in a variety of critical illnesses, including ALI and ARDS. Among available specialty formulas, the so-called "immunoenhancing" formulas, which are supplemented with arginine, have been extensively evaluated in clinical studies. Although debate continues regarding a potential role for immunoenhancing diets in critical illness, most authors agree that after more than 20 years of research involving thousands of patients, immunoenhancing diets should not be routinely used in

\footnotetext{
${ }^{* 1}$ Department of Nutrition and Intensive Care, Fernandes Távora Hospital

Rua Ildefonso Albano 777/403, Fortaleza, Ceará 60.115-000, Brazil Accepted
$* 2$ Department of Emergency and Critical Care Medicine, Chiba University Graduate School of Medicine

Submitted for publication November 10, 2009 Accepted for publication June 15, 2010 1-8-1 Inohana, Chuo-ku, Chiba, Chiba 260-8677, Japan
} 
this population. Moreover, the currently available evidence suggests that these diets may be associated with increased mortality rates in patients with systemic inflammatory diseases, such as sepsis ${ }^{4)}$.

On the other hand, a new type of enteral formula enhanced with special anti-inflammatory lipids showed promise in the treatment of critical illness. Specifically, a formula supplemented with $\gamma$-linolenic acid (GLA), an $\Omega$-6 lipid derived from borage oil, and eicosapentaenoic acid (EPA), an $\Omega-3$ lipid derived from fish oil, together with enhanced levels of antioxidant vitamins (mainly vitamins $\mathrm{C}$ and $\mathrm{E}$ ) and containing no added arginine, has recently been found to decrease morbidity and also mortality in critically ill, mechanically ventilated patients suffering from ALI and ARDS 5) 7). This type of formula appears to inhibit systemic inflammation through a decrease in the levels of arachidonic acid (AA), an $\Omega-6$ lipid involved in the production of various proinflammatory eicosanoids such as prostaglandin $\mathrm{E}_{2}$ $\left(\mathrm{PGE}_{2}\right)$ and thromboxane $\mathrm{A}_{2}\left(\mathrm{TXA}_{2}\right)^{8)}$. High levels of these AA-derived pro-inflammatory eicosanoids can produce systemic inflammation, enhanced chemotaxis and platelet aggregation, as well as microvascular thrombosis and immune suppression. EPA and GLA help to decrease this inflammation by rebalancing proinflammatory and anti-inflammatory eicosanoid production ${ }^{9}$. In a recent systematic review of immunonutrition in critically ill patients, Marik and Zaloga indicated that only the enteral formula enriched with EPA, GLA and antioxidant vitamins improved the outcome of medical ICU patients with systemic inflammatory response syndrome (SIRS)/sepsis/ARDS ${ }^{10)}$.

In many institutions, an enteral formula enriched with EPA, GLA and antioxidant vitamins has emerged as the standard of care for patients with acute lung pathologies. Due to its well described anti-inflammatory properties and the absence of added arginine, the new designation of "inflammation-modulating diet" has been proposed to identify this new class of specialized formula and to set it apart from the immunoenhancing formulas ${ }^{11), 12)}$.

Three independently conducted, prospective, randomized controlled studies were performed using a diet enriched with EPA, GLA, and antioxidant vitamins in different populations suffering from ALI/ARDS 5) 7). These 3 studies associated the use of an enteral diet enriched with EPA + GLA + antioxidant vitamins with important improvements in ICU outcomes including improvements in the $\mathrm{P} / \mathrm{F}$ ratio (oxygenation status), more ventilator-free days, more ICU-free days, less development of new organ failures and lower mortality rates. On the other hand, it has been reported that the efficacy of nutritional treatment is affected by the genetic conditions related to metabolism and nutrition such as the presence of genetic polymorphisms, and such concept is referred to as nutrigenomics ${ }^{13), 14)}$. It is also reported that there is ethnic difference in the distribution of genetic polymorphisms ${ }^{15}$ ). Therefore, the efficacy of the diet enriched with EPA, GLA, and antioxidant vitamins might be different in Japanese populations from that in other populations even though the diet was proved to be very effective on general Brazilian populations ${ }^{7}$. Since one of the trials ${ }^{7)}$ was conducted in Brazil, which has one of the largest Japanese colonies in the world, this study aims to evaluate the effects of this enteral nutrition strategy on a population of Japanese-descendant patients with ARDS associated with severe sepsis or septic shock through a retrospective assessment of the original database of the Brazilian study.

\section{Materials and methods}

Study design and ethical aspects: This study represents a retrospective assessment of the database of a prospective, single-center, randomized, double-blinded, controlled trial comparing the effects of an enteral nutrition enriched with EPA, GLA and elevated levels of antioxidant vitamins with an isonitrogenous and isocaloric control diet in patients diagnosed with either severe sepsis or septic shock and requiring mechanical ventilation. Patients were recruited from 3 different adult intensive care units (one medical, one cardiologic and one post-surgical ICU) of a single tertiary hospital during a 15-month period.

This study was approved by the Institutional Review Board before patient registration. Informed consent was obtained from all patients or their legal representatives. All patients were treated in accordance with the Surviving Sepsis Campaign guidelines for management of severe sepsis and septic shock ${ }^{16)}$. Patients received steroids when they remained hypotensive despite an adequate fluid replacement in a total of 200-300 mg/day of hydrocortisone for 7 days in 4 divided doses. Adequate initial resuscitation was given to all patients with sepsisinduced tissue hypoperfusion in accordance with the protocol of Rivers, et al. ${ }^{17)}$ Adequate broad spectrum antibiotics were also delivered to all patients in accordance with the current guidelines ${ }^{16)}$.

Study patients: Patients were enrolled under the conditions of being over 18 years of age, requiring mechanical ventilation (with a maximum $\mathrm{P} / \mathrm{F}$ ratio $<200$, no minimum value was established), having enteral access, and a clinical diagnosis of either severe sepsis or septic shock, according to the criteria defined by Bone, et al. ${ }^{18)}$ and modified by Bernard, et al. ${ }^{19)}$ Cardiovascular, renal, hematologic, hepatic and neurologic failures were defined by previously published criteria ${ }^{19}$ ). Gastrointestinal failure was assessed using the criteria previously defined by Gadek, et $\mathrm{al}^{5}$.

The main reasons for exclusion were: pregnancy or breastfeeding, less than 18 years of age, significant limitation of survival prognosis (defined as a lifeexpectancy below 28 days, due to a chronic and/or incurable disease such as uncontrolled cancer or other terminal disease), pre-existing chronic renal insufficiency (acute renal insufficiency was not considered an exclusion criteria), acute pancreatitis without established origin, participation in another clinical trial less than 30 days before, head trauma with a Glasgow coma scale $(\mathrm{GCS}) \leq 5$, recent stroke or subarachnoid hemorrhage, 
important immunologic suppression (defined as a leukocyte count below 5,000 cells $/ \mathrm{mm}^{3}$ ), infection by $\mathrm{HIV}$, no indication for enteral nutrition or imminence of receiving parenteral nutrition, receiving partial parenteral nutrition, presence of uncontrolled diarrhea, recent gastrointestinal bleeding event, planned weaning from mechanical ventilation prior to Study Day 4, and exclusion from the protocol by physicians' decision. Eighteen patients from the original database were identified as Japanese-descendant patients. The criteria used to define the patient as a Japanese-descendant was that of a positive answer to ethnic origin collected on the clinical research form (CRF) in the original study. Baseline demographic and clinical characteristics of the 18 patients included in this re-assessment are shown in Table 1.

Randomization and study diet administration: Patients were randomized in a ratio of $1: 1$ in a blinded way. The control diet was a high-fat, low carbohydrate enteral formula, balanced for patients with pulmonary diseases. The study diet was enriched with EPA, GLA, and antioxidant vitamins, though it remained isocaloric and isonitrogenous to the control diet. The full composition of the two diets is shown in Table 2. Patients received enteral nutrition within 6 hours of meeting entry criteria. Enteral feeding was delivered at a constant rate to achieve a minimum of $50 \%$ of basal energy expenditure (BEE; determined using the Harris-Benedict equation) $x 1.3$ within the first 24 hours ${ }^{20)}$. If well tolerated, enteral nutrition was advanced to achieve a minimum of $75 \%$ of BEE x 1.3 within 72 hours. The enteral diets were delivered until patients were extubated, or until interrupted at the physician's discretion or due to the development of any adverse event that could be related to the enteral feeding.

The day on which patients fulfilled all entry criteria was considered baseline and the first day the patient received a minimum of $75 \%$ of $B E E \times 1.3$ was considered Study Day 1. Enteral diet was delivered continuously for a minimum of 4 study days at a rate not exceeding BEE x 1.3. The daily enteral intake was recorded in order to obtain the total volume and calories delivered to the patient.

Laboratory data, outcome measures and statistical analysis: Blood samples were obtained for routine laboratory testing at least on baseline, and on Study Days 4 and 7 . The $\mathrm{P} / \mathrm{F}$ ratio was assessed at least daily during the feeding period. A simultaneous recording of ventilator settings was also performed. Weaning from mechanical ventilation was performed using the protocol previously described by Esteban, et al. ${ }^{21)}$

The primary endpoint was to determine the mortality rate from any cause during a 28-day follow-up period. Secondary endpoints included changes in oxygenation status, time receiving mechanical ventilation, period in the ICU, and development of new organ dysfunctions.

Oxygenation status was determined by the $\mathrm{P} / \mathrm{F}$ ratio. Time receiving mechanical ventilation and period in the ICU were defined as the number of days from study entry
Table 1 Baseline demographic and clinical characteristics of the two groups ${ }^{7)}$

\begin{tabular}{lcc}
\hline \hline \multicolumn{1}{c}{ Characteristic } & $\begin{array}{c}\text { Control diet } \\
(\mathrm{n}=8)\end{array}$ & $\begin{array}{c}\text { EPA + GLA } \\
\text { enriched diet } \\
(\mathrm{n}=10)\end{array}$ \\
\hline Age & $62.4 \pm 17.2$ & $65.6 \pm 12.7$ \\
Gender & 5 & 7 \\
$\quad$ Male & 3 & 3 \\
$\quad$ Female & & \\
Type of enteral feeding & 6 & 7 \\
$\quad$ Gastric & 2 & 2 \\
$\quad$ Duodenal & 0 & 1 \\
$\quad$ Jejunal & $62.8 \pm 4.3$ & $60.5 \pm 2.7$ \\
Admission weight $(\mathrm{kg})$ & $9.0 \pm 0.8$ & $8.7 \pm 0.3$ \\
SOFA score & $3.7 \pm 0.5$ & $3.1 \pm 0.4$ \\
Serum albumin $(\mathrm{g} / \mathrm{l})$ & $1.2 \pm 0.4$ & $0.9 \pm 0.3$ \\
Serum creatinine $(\mathrm{mg} / l)$ &
\end{tabular}

SOFA, sequential organ failure assessment.

(baseline) to the actual day that a patient remained on a ventilator or in the ICU, respectively, during the 28-day follow-up period. New organ dysfunction was defined as the development of any new organ failure (cardiovascular, renal, hematologic, hepatic, neurologic, and gastrointestinal) during the 28-day follow-up period that had not been present at time of study entry (baseline), or the development of a new respiratory failure requiring new ventilatory support. The criteria used to determine new organ failures were the same as those used to determine baseline dysfunctions.

A patient was considered evaluable if : baseline measures of oxygenation were obtained; a feeding period of 4 days from Study Day 1 was realized; all clinical measures of oxygenation were available, and the patient was monitored until the end of the 28-day follow-up period or when death occurred.

Differences were assessed using the chi-square test, or Fisher's exact test if appropriate for categorical variables, and the Student's $t$-test for continuous variables ${ }^{22)}$.

Estimates of survival curves were calculated using the Kaplan-Meier product-limit method ${ }^{23)}$ and compared by using the log-rank test ${ }^{24)}$. The Newcombe-Wilson method without continuity correction was used to calculate a confidence interval for the difference between two proportions ${ }^{25)}$. Confidence intervals for relative risk were calculated using the methods described by Armitage and Berry ${ }^{26)}$.

One-way analysis of covariance (ANOVA) with repeated measures was used to provide comparisons among groups of the changes in the response variable from baseline at each of the successive measurements.

All $P$ values were two-tailed. A $P<0.05$ was considered statistically significant. All computations were performed using SAS v. 8.2 software (SAS Institute Inc., Cary, NC, USA) and Minitab v. 14.1 (Minitab Inc., State College, PA, USA). All data are expressed as mean \pm SE 
日集中医誌 J Jpn Soc Intensive Care Med Vol. 18 No. 2

Table 2 Composition of the enteral diets ${ }^{7)}$

\begin{tabular}{|c|c|c|c|c|c|}
\hline Nutrient & Control diet & $\begin{array}{l}\text { EPA + GLA } \\
\text { enriched diet }\end{array}$ & Nutrient & Control diet & $\begin{array}{l}\text { EPA + GLA } \\
\text { enriched diet }\end{array}$ \\
\hline Protein & & & Vitamin D (IU/l) & 423 & 430 \\
\hline$\%$ of total calories & 16.7 & 16.7 & Vitamin $\mathrm{K}_{1}(\mu \mathrm{g} / l)$ & 85 & 100 \\
\hline $\mathrm{g} / l$ & 62.6 & 62.5 & Folic acid $(\mu \mathrm{g} / l)$ & 845 & 420 \\
\hline \multirow[t]{2}{*}{ Source } & $87 \%$ sodium caseinate & $87 \%$ sodium caseinate & Thiamine (mg/l) & 3.2 & 3.2 \\
\hline & $13 \%$ calcium caseinate & $13 \%$ calcium caseinate & Riboflavin (mg/l) & 3.6 & 3.6 \\
\hline Carbohydrate & & & Vitamin $\mathrm{B}_{6}(\mathrm{mg} / \mathrm{l})$ & 4.2 & 4.3 \\
\hline$\%$ of total calories & 28.1 & 28.1 & Vitamin $B_{12}(\mathrm{mg} / \mathrm{l})$ & 13 & 6.0 \\
\hline $\mathrm{g} / l$ & 102.0 & 105.5 & Niacin $(\mathrm{mg} / \mathrm{l})$ & 42 & 29 \\
\hline \multirow[t]{2}{*}{ Source } & $46 \%$ maltodextrin & $45 \%$ maltodextrin & Choline $(\mathrm{mg} / \mathrm{l})$ & 630 & 640 \\
\hline & $54 \%$ sucrose & $55 \%$ sucrose & Biotin $(\mu \mathrm{g} / l)$ & 630 & 1 \\
\hline Lipids & & & Panthothenic acid & 22 & 13 \\
\hline$\%$ of total calories & 55.2 & 55.2 & $(\mathrm{mg} / \mathrm{l})$ & & \\
\hline $\mathrm{g} / l$ & 93.4 & 93.7 & Trace minerals & & \\
\hline \multirow[t]{5}{*}{ Source } & $55.8 \%$ canola oil & $31.8 \%$ canola oil & $\mathrm{Na}(\mathrm{mg} / \mathrm{l})$ & 1,310 & 1,310 \\
\hline & $14 \%$ corn oil & $25 \% \mathrm{MCT}$ & $\mathrm{K}(\mathrm{mg} / \mathrm{l})$ & 2,000 & 1,960 \\
\hline & $20 \% \mathrm{MCT}$ & $20 \%$ borage oil & $\mathrm{Cl}(\mathrm{mg} / \mathrm{l})$ & 1,690 & 1,690 \\
\hline & $7 \%$ high oleic safflower oil & $20 \%$ fish oil & $\mathrm{Ca}(\mathrm{mg} / \mathrm{l})$ & 1,060 & 1,060 \\
\hline & $3.2 \%$ soy lecithin & $3.2 \%$ soy lecithin & $\mathrm{P}(\mathrm{mg} / \mathrm{l})$ & 1,060 & 1,000 \\
\hline$\Omega 6: \Omega 3$ & $3.8: 1$ & $1.85: 1$ & $\mathrm{Mg}(\mathrm{mg} / \mathrm{l})$ & 426 & 320 \\
\hline$\Omega 3(\mathrm{~g} / \mathrm{l})$ & 0.35 & 10 & $\mathrm{I}(\mu \mathrm{g} / l)$ & 160 & 160 \\
\hline $\mathrm{EPA}(\mathrm{g} / l)$ & 0 & 4.5 & $\operatorname{Mn}(\mathrm{mg} / \mathrm{l})$ & 5.3 & 5.4 \\
\hline GLA $(\mathrm{g} / l)$ & 0 & 4.3 & $\mathrm{Cu}(\mathrm{mg} / \mathrm{l})$ & 2.2 & 2.2 \\
\hline DHA $(\mathrm{g} / l)$ & 0 & 2.0 & $\mathrm{Zn}(\mathrm{mg} / \mathrm{l})$ & 24 & 18 \\
\hline Vitamins & & & $\mathrm{Fe}(\mathrm{mg} / \mathrm{l})$ & 19 & 20 \\
\hline Vitamin E (IU/l) & 65 & 320 & $\operatorname{Se}(\mu \mathrm{g} / l)$ & 80 & 130 \\
\hline Vitamin C (mg/l) & 317 & 840 & $\mathrm{Cr}(\mu \mathrm{g} / l)$ & 127 & 160 \\
\hline B-carotene $(\mathrm{mg} / \mathrm{l})$ & 647 & 670 & $\operatorname{Mo}(\mu \mathrm{g} / l)$ & 165 & 130 \\
\hline Taurine $(\mathrm{mg} / \mathrm{l})$ & 160 & 320 & Caloric density $(\mathrm{kcal} / \mathrm{m} l)$ & 1.5 & 1.5 \\
\hline L-carnitine $(\mathrm{mg} / \mathrm{l})$ & 160 & 120 & Osmolarity $(\mathrm{mOsm} / \mathrm{l})$ & 372 & 385 \\
\hline Vitamin A (IU/l) & 11,800 & 12,000 & & & \\
\hline
\end{tabular}

DHA, docosahexaenoic acid; EPA, eicosapentaenoic acid; GLA, $\gamma$-linolenic acid; IU, international units; MCT, medium chain triglycerides.

or as indicated.

\section{Results}

Study groups: A total of 165 patients were enrolled in the original study, of which 18 patients were considered as evaluable Japanese-descendants. There were no significant differences with respect to the delay in intensive care admission, or rates of preexisting diabetes, hyperglycemia and hypertension. There are no statistically significant differences between the groups in relation to the use of corticoids.

The mean dietary intake was similar between groups for total calories $(1,597 \pm 32$ vs. $1,618 \pm 27 \mathrm{kcal} / 24$ hours, $P>0.5$; study vs. control group).

The average time from study entry to the beginning of the enteral diet was similar between the study and control groups $(3.1 \pm 0.6$ vs. $3.4 \pm 0.7$ hours respectively, $P>$ 0.5 ). Average time to achieve $75 \%$ of BEE x 1.3 (Study Day 1) was also similar $(27.2 \pm 3.9$ vs. $27.9 \pm 2.4$ hours).
Respiratory gas exchange: There were no statistically significant differences in $\mathrm{PaO}_{2}, \mathrm{FIO}_{2}$, and $\mathrm{P} / \mathrm{F}$ ratio between the groups at baseline. All patients included in this study had severely compromised oxygenation status, placing them in the ARDS category in accordance with the consensus definition of a $\mathrm{P} / \mathrm{F}$ ratio of $<200^{27}$ ). The $\mathrm{P} / \mathrm{F}$ ratio remained unchanged for patients receiving the control diet until Study Day 7. In contrast, patients receiving the study diet had statistically significant increases in P/F ratios on Study Days 4 and 7, depicted in Fig. 1. All patients who were extubated between Study Day 4 and Study Day 7 were excluded from this analysis.

No differences were observed in baseline $\mathrm{P} / \mathrm{F}$ ratio between the two groups $(155.4 \pm 10.2$ vs. $149.1 \pm 11.7$, $P=0.6916$, study vs. control group). However, patients fed the study diet increased their $\mathrm{PaO}_{2}$ when compared with the control group on Study Day 4, and maintained this improvement until Study Day 7.

ICU and ventilator outcomes: Patients fed the study diet had more ventilator-free days when compared to 


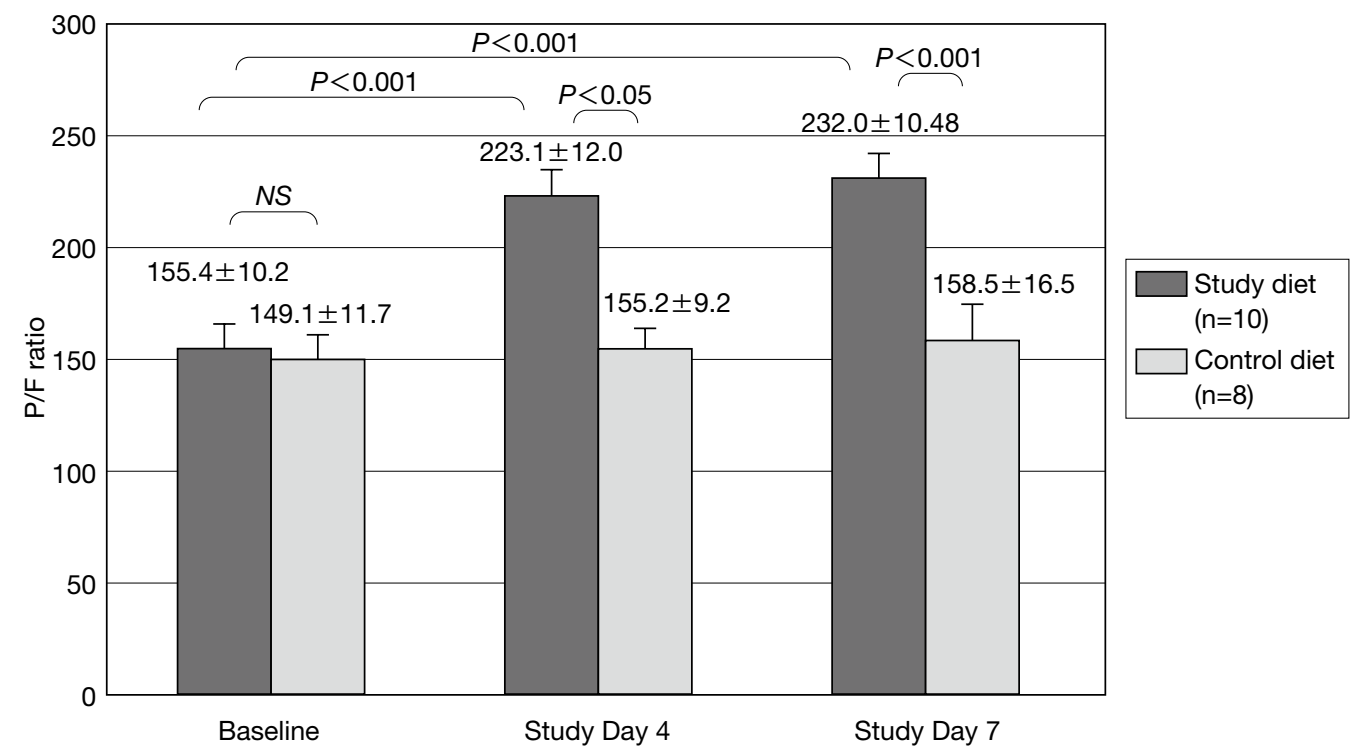

Fig. 1 Improvement of oxygenation status of patients fed EPA + GLA when compared to patients receiving control diet ${ }^{7)}$

EPA, eicosapentaenoic acid; GLA, $\gamma$-linolenic acid.

(a)

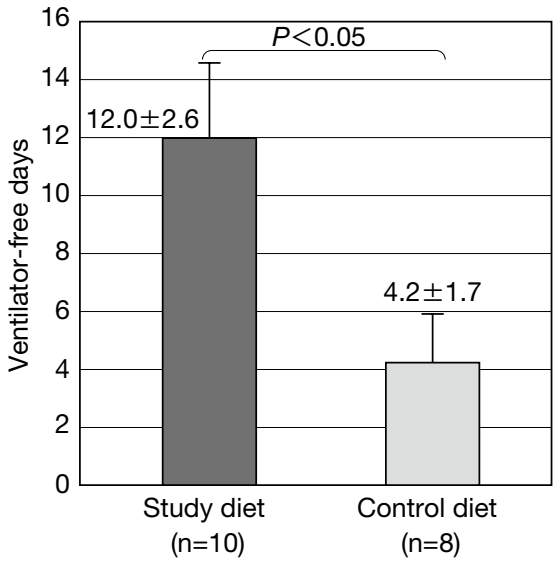

(b)

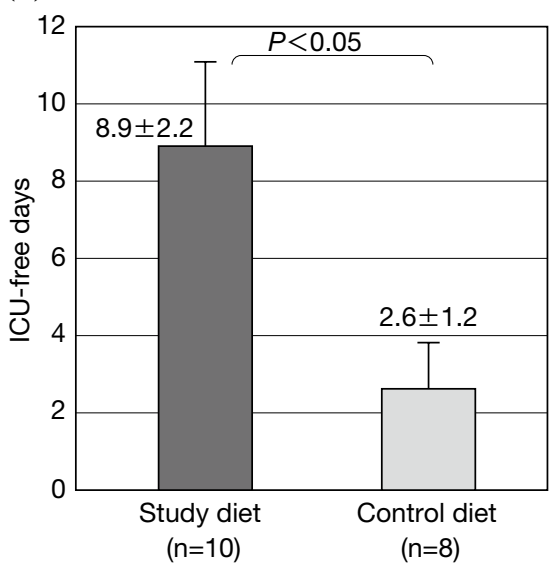

Fig. 2 Mean \pm SE number of 28-day ventilator-free days and ICU-free days ${ }^{7)}$

(a) Patients fed the enteral diet enriched with EPA + GLA had 7.7 more ventilator-free days $(P=0.0362)$ when compared with patients fed the control diet.

(b) Patients fed the enteral diet enriched with EPA + GLA had 6.2 more ICU-free days $(P=0.0348)$ when compared with patients fed the control diet.

EPA, eicosapentaenoic acid; GLA, $\gamma$-linolenic acid; SE, standard error of mean.

patients fed the control diet $(12.0 \pm 2.6$ vs. $4.2 \pm 1.7$, respectively, $P=0.0362$ ) (Fig. 2a). In addition, patients fed the study diet had more ICU-free days than patients fed the control diet $(8.9 \pm 2.2$ vs. $2.6 \pm 1.2$, respectively, $P=0.0348$ ) (Fig. 2b).

The development of new organ dysfunction was also analyzed but the results were considered non-significant. Patients fed with the study diet developed $1.4 \pm 0.6$ new organ dysfunctions, and patients fed the control diet developed $2.6 \pm 0.8$ new organ dysfunctions during the 28-day follow-up period $(P>0.5)$.

Mortality: On Day 28, there were 3 deaths (30\%), in the group that received the study diet, compared to 4 deaths $(50 \%)$ in the control diet, and this difference was considered non-significant $(P>0.5)$ in accordance with the log-rank test.

\section{Discussion}

This study confirmed previously published results demonstrating the beneficial effects of an enteral diet enriched with EPA, GLA and antioxidant vitamins, on patients with ARDS ${ }^{5)}$. The use of this diet in a Japanesedescendant population of patients suffering from ARDS associated with severe sepsis or septic shock was retrospectively associated with an improvement in oxygenation status, reduced mechanical ventilation time and 
period in the ICU. The diet used was an isonitrogenous and isocaloric enteral formula, differing from the control diet only in its lipid composition and level of antioxidant vitamins. Since there is evidence in the literature pointing towards the anti-inflammatory roles not only of EPA and GLA ${ }^{28)}$, but also of antioxidant vitamins alone 29) 31), the differences between both groups may be explained not just by the effects of EPA, GLA or antioxidant vitamins, but also by a combination of them.

One of the main characteristics of this trial is that it only allows 6 hours from the moment at which patients fulfilled all entry requirements to effective onset of diet, rather than 24 hours, leading to a significant reduction in time necessary to achieve $75 \%$ of BEE x 1.3. Recent studies have shown that time-dependence is a determinant aspect in the treatment of septic patients.

For instance, the recombinant human activated Protein C Worldwide Evaluation in Severe Sepsis (PROWESS) study ${ }^{32)}$ showed a significant reduction in the mortality of severe septic patients with a high Acute Physiology and Chronic Health Evaluation (APACHE) II score who were treated with recombinant human activated protein $\mathrm{C}$ (rhAPC) within the first 48 hours after fulfillment of the study entry criteria. Nevertheless, the Extended Evaluation of Recombinant Human Activated Protein C (ENHANCE) study showed that septic patients who were treated with rhAPC within the first 24 hours after meeting the inclusion criteria had lower mortality than those patients who were treated after 24 hours, but within the first 48 hours ${ }^{33}$ ). The early use of rhAPC was also associated with a lower consumption of hospital resources including mechanical ventilation and the use of vasopressors ${ }^{33}$ ). Time-dependency was also associated with several other recommendations for the management of septic patients ${ }^{16)}$.

Regarding oxygenation status, a significant improvement was found in patients receiving the study diet. Such improvement was already observed by Day 4, and remained until Day 7, different from patients who received the control diet in whom no significant improvement in oxygenation status was found.

The respiratory tract is the most common site of infection in septic patients ${ }^{32), 34)}$. Studies have demonstrated that over $70 \%$ of critical septic patients require mechanical ventilation ${ }^{19)}$, suggesting that the pulmonary inflammatory process has a fundamental role in regulating the systemic inflammatory response, which may lead the patient to a septic state or sustain this state of inflammatory hyperactivity. Patients fed with EPA, GLA, and antioxidant vitamins during inflammatory diseases, such as ARDS, showed significant reduction in recruitment of pulmonary neutrophils and lower level of pro-inflammatory mediators ${ }^{35)}$. This is an important aspect since the mass inflammatory response is the most common path in ARDS and sepsis pathogenesis. An enternal diet enriched with EPA + GLA + antioxidant vitamins may assist in the restoration of the balance between pro- and anti-inflammatory mediators by modulating the metabolism of arachidonic acid and also through synthesis of anti-inflammatory eicosanoids ${ }^{35)}$.

Furthermore, the decrease in both mechanical ventilation days and period in the ICU, amongst the population of patients that used the study diet, indicates lower use of available resources. An important characteristic of this approach relates to the price of such an intervention, which is relatively low compared with other forms of treatment of the septic patient. In addition, this therapeutic intervention may be considered very safe.

If we understand that patients with sepsis are under a persistent and uncontrolled state of inflammation, wherein the inflammatory reaction is capable of spreading indefinitely, leading the patient to severe shock, multiple organ failure and death ${ }^{36)}$, strategies for treatment capable of modulating this chain of inflammatory events may be worth further investigation. One limitation to the findings in this assessment is the small sample size. It is important to consider that this can be a potential source of Type I error (false positive).

The result of the present study indicates that the diet enriched with EPA+GLA and antioxidant vitamins is equally effective in a Japanese-descendant population in Brazil who probably have the same distribution of genetic polymorphisms as Japanese living in Japan compared to the effect in the general Brazilian population.

\section{Conclusions}

This study shows the clinical efficacy of a lowcarbohydrate, high-fat enteral formula containing an oil blend of EPA and GLA with elevated antioxidant vitamins in a Japanese-descendant population of critically ill patients with severe sepsis or septic shock, requiring mechanical ventilation and tolerating enteral nutrition. Our results suggest that this enteral formula can contribute to better ICU outcomes even in a Japanese population living in Japan.

\section{References}

1) DeMichele SJ, Wood SM, Wennberg AK. A nutritional strategy to improve oxygenation and decrease morbidity in patients who have acute respiratory distress syndrome. Respir Care Clin 2006;12:547-66.

2) Rubenfeld GD, Caldwell E, Peabody E, et al. Incidence and outcomes of acute lung injury. N Engl J Med 2005;353: 1685-93.

3) Mutlu GM, Budinger GR. Incidence and outcomes of acute lung injury. N Engl J Med 2006;354:416-7.

4) Heyland DK, Dhaliwal R. Immunonutrition in the critically ill: from old approaches to new paradigms. Intensive Care Med 2005;31:501-3

5) Gadek JE, DeMichele SJ, Karlstad MD, et al. Effect of enteral feeding with eicosapentaenoic acid, $\gamma$-linolenic acid and antioxidants in patients with acute respiratory distress syndrome. Crit Care Med 1999;27:1409-20.

6) Singer P, Theilla M, Fisher H, et al. Benefit of an enteral diet enriched with eicosapentaenoic acid and $\gamma$-linolenic acid in ventilated patients with acute lung injury. Crit Care Med 2006;34:1033-8.

7) Pontes-Arruda A, Aragão AM, Albuquerque JD. Effects of enteral feeding with eicosapentaenoic acid (EPA), $\gamma$-linolenic acid and antioxidants on mechanically ventilated patients with 
severe sepsis and septic shock. Crit Care Med 2006;34: 2325-33.

8) Mancuso P, Whelan J, DeMichele SJ, et al. Dietary fish oil and borage oil suppress intrapulmonary proinflammatory eicosanoids biosynthesis and attenuate pulmonary neutrophil accumulation in endotoxic rats. Crit Care Med 1997;25:1198206.

9) Ziboh VA, Fletcher MP. Dose-response effects of dietary $\gamma$-linolenic acid-enriched oils on human polymorphonuclearneutrophil biosynthesis of leukotriene $\mathrm{B}_{4}$. Am J Clin Nutr 1992;55:39-45.

10) Marik PE, Zaloga GP. Immunonutrition in critically ill patients: a systematic review and analysis of the literature. Intensive Care Med 2008;34:1980-90.

11) Pontes-Arruda A. The use of special lipids in the treatment of inflammatory lung disease. Clin Nutr Insight 2007;33:1-4.

12) Martindale RG, McClave SA, Vanek VW, et al. Guidelines for the provision and assessment of nutrition support therapy in the adult critically ill patient: Society of Critical Care Medicine and American Society for Parenteral and Enteral Nutrition: Executive Summary. Crit Care Med 2009;37:1757-61.

13) Gillies PJ. Nutrigenomics: the Rubicon of molecular nutrition. J Am Diet Assoc 2003;103:S50-5.

14) Ghosh D, Skinner MA, Laing WA. Pharmacogenomics and nutrigenomics: synergies and differences. Eur J Clin Nutr 2007;61:567-74.

15) Watanabe E, Hirasawa H, Oda S, et al. Cytokine-related genotypic differences in peak interleukin-6 blood levels of patients with SIRS and septic complications. J Trauma 2005;59:1181-90.

16) Dellinger RP, Carlet JM, Masur H, et al. Surviving Sepsis Campaign guidelines for management of severe sepsis and septic shock. Crit Care Med 2004;32:858-73.

17) Rivers E, Nguyen B, Havstad S, et al. Early goal-directed therapy in the treatment of severe sepsis and septic shock. $\mathrm{N}$ Engl J Med 2001;345:1368-77.

18) Bone RC, Balk RA, Cerra FB, et al. ACCP/SCCM Consensus Conference. Definitions for sepsis and organ failure and guidelines for the use of innovative therapies in sepsis. Chest 1992;101:1644-55.

19) Bernard GR, Margolis BD, Shanies HM, et al. Extended evaluation of recombinant human activated protein $\mathrm{C}$ United States trial (ENHANCE US). A single-arm, phase 3B, multicenter study of drotrecogin alfa (activated) in severe sepsis. Chest 2004;125:2206-16.

20) Donaldson-Andersen J, Fitzsimmons L. Metabolic requirements of the critically ill, mechanically ventilated trauma patients: measured versus predicted energy expenditure. Nutr Clin Pract 1998;13:25-31.

21) Esteban A, Alia I, Tobin MJ, et al. Effect of spontaneous breathing trial duration on outcome of attempts to discontinue mechanical ventilation. Spanish Lung Failure Collaborative Group. Am J Respir Crit Care Med 1999;159:512-8.

22) Rosner B. Fundamentals of biostatistics. 2nd ed. Boston: PWS Publishers; 1986. p. 584.

23) Kaplan EL, Meier P. Nonparametric estimation from incomplete observations. J Am Stat Assoc 1958;53:457-81.

24) Mantel N. Evaluation of survival data and two new rank order statistics arising in its consideration. Cancer Chem Rep 1966; 50:163-70

25) Newcombe RG. Interval estimation for the difference between independent proportions: Comparison of eleven methods. Stat Med 1998;17:873-90.

26) Armitage P, Berry G. Statistical methods in medical research. 3rd ed. London: Blackwell; 1994. p. 131.

27) Bernard GR, Artigas A, Brigham KL, et al. The AmericanEuropean Consensus Conference on ARDS. Definitions, mechanisms, relevant outcomes, and clinical trial coordination. Am J Respir Crit Care Med 1994;149:818-24.

28) Palombo JD, DeMichele SJ, Boyce PJ, et al. Effect of shortterm enteral feeding with eicosapentaenoic and $\gamma$-linolenic acids on alveolar macrophage eicosanoid synthesis and bacterial function in rats. Crit Care Med 1999;27:1908-15.

29) Bulger EM, Helton WS, Clinton CM, et al. Enteral vitamin E supplementation inhibits the cytokine response to endotoxin. Arch Surg 1997;132:1337-41.

30) Seeger W, Ziegler A, Wolf HR. Serum alpha-tocopherol levels after high-dose enteral vitamin $\mathrm{E}$ administration in patients with acute respiratory failure. Intensive Care Med 1987;13:395400.

31) Bulger EM, Jurkovich GJ, Gentilello LM, et al. Current clinical options for the treatment and management of acute respiratory distress syndrome. J Trauma 2000;48:562-72.

32) Bernard GR, Vincent JL, Laterre PF, et al. Efficacy and safety of recombinant human activated protein $\mathrm{C}$ for severe sepsis. $\mathrm{N}$ Engl J Med 2001;344:699-709.

33) Vincent JL, Bernard GR, Beale R, et al. Drotrecogin alfa (activated) treatment in severe sepsis from the global open-label trial ENHANCE: further evidence for survival and safety and implications for early treatment. Crit Care Med 2005;33: 2266-77.

34) Silva E, Pedro Mde A, Sogayar AC, et al. Brazilian sepsis epidemiological study (BASES Study). Crit Care 2004;8:R251-60.

35) Pacht ER, DeMichele SJ, Nelson JL, et al. Enteral nutrition with eicosapentaenoic acid, $\gamma$-linolenic acid and antioxidants reduces alveolar inflammatory mediators and protein influx in patients with acute respiratory distress syndrome. Crit Care Med 2003;31:491-500.

36) Bone RC. Sir Isaac Newton, sepsis, SIRS and CARS. Crit Care Med 1996;24:1125-8. 